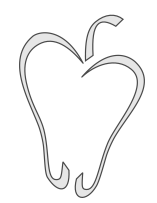

ISSN 1852-4249

\title{
Invited review: Graphite and its hidden superconductivity
}

\author{
P. Esquinazi ${ }^{1 *}$
}

\begin{abstract}
We review experimental results, from transport to magnetization measurements, on different graphite samples, from bulk oriented graphite, thin graphite films to transmission electron microscope lamellae, that indicate the existence of granular superconductivity at temperatures above $100 \mathrm{~K}$. The accumulated evidence speaks for a localization of the superconducting phase(s) at certain interfaces embedded in semiconducting crystalline regions with Bernal stacking order.
\end{abstract}

\section{Introduction}

Over the past decade, our interpretation of the magnetic and transport properties of ordered graphite bulk samples has experienced a change respect to the partially accepted general description of their intrinsic properties. The description of graphite one finds in the not-so-old literature tells us that it is a kind of (semi)metal with a finite Fermi energy and carrier (electron plus hole) densities per graphene layer at low temperatures $n_{0} \sim 10^{10} \ldots 10^{12} \mathrm{~cm}^{-2}$, see e.g., [1-3]. However, real samples are not necessarily ideal, we mean defect-free, and therefore those carrier densities are not necessarily intrinsic of ideal graphite. The exhaustive experience accumulated in gapless and narrow band semiconductors [4] already indicates us how important defects and impurities (not necessarily magnetic ones but, for example, hydrogen) are in determining some of the measured properties. Therefore, taking experimental data of real samples as intrinsic, without knowing their microstructure

*E-mail: esquin@physik.uni-leipzig.de

1 Division of Superconductivity and Magnetism, Institute for Experimental Physics II, Fakultät für Physik und Geowissenschaften, Universität Leipzig, Linnéstrasse 5, D04103 Leipzig, Germany. and/or defect concentration, was indeed a misleading assumption in the past. This assumption has drastically influenced the description of the band structure of graphite we found nowadays in several books and publications. For example, if graphite has a finite Fermi energy $E_{F}$ (whatever the majority carriers are), as assumed everywhere, up to seven free parameters have to be introduced $[2,5,6]$ to describe the apparently ideal band structure of Bernal graphite with the well-known ABAB stacking order of the graphene layers.

The impact of well defined two-dimensional interfaces inside graphite samples $[7,8]$ had not been realized until recent studies of the transport properties as a function of thickness of the graphite sample provided a link to the microstructure of the samples obtained by transmission electron microscope (TEM) studies. We also have to add the sensitivity of the graphite transport properties to very small amount of defects [9]. Those results [7,9] do not only indicate us that at least a relevant part of the carrier densities measured in graphite is not intrinsic but also that the metallic-like behavior of the electrical resistance does not reflect ideal, defect-free graphite [10]. An anomalous vanishing of the amplitude of the Shubnikov-de Haas $(\mathrm{SdH})$ oscillations decreasing the thickness of the graphite samples was published, more than 10 years 
Papers in Physics, vol. 5, ART. 050007 (2013) / P. Esquinazi

ago, [11] without attracting the necessary attention, although those results already suggested that the $\mathrm{SdH}$ oscillations are probably not intrinsic of the graphite structure. These results are supported by the absence of $\mathrm{SdH}$ oscillations, i.e., no evidence for the existence of a Fermi surface, found recently in bulk oriented samples of high grade and high purity but without internal interfaces [12]. All these results indicate that the internal microstructure of the graphite samples play an important role, a microstructure that was neither characterized nor considered in the discussion of the measured properties of different graphite samples, from highly oriented pyrolytic graphite (HOPG) to Kish or natural graphite, even in nowadays literature $[6,13,14]$.

What does this have to do with superconductivity? If we start searching for superconductivity in graphite by measuring the behavior of the electrical resistance $(R)$ with temperature $(T)$ and magnetic field $(H)$, for example, it should be clear that the knowledge of the intrinsic, normal state dependence is needed. Otherwise, we may misleadingly interpret an anomalous behavior due to, for example, the influence of non-percolative, granular superconducting regions embedded in a (normal state) graphite matrix, as intrinsic of the material, clearly missing an interesting aspect of the sample. A reader with expertise in superconductivity might not be convinced that such a mistake could be ever made. However, the ballistic transport characteristics of the graphene layers in ideal graphite with their huge mobility and mean free path [15-18] provide a high conductivity path in parallel; such that it is not at all straightforward by simple experiments to realize and prove the existence of superconductivity at certain regions in some, not all, graphite samples. One needs indeed to do systematic experiments decreasing the size of the graphite samples (but not too much) to obtain clear evidence for the embedded or "hidden" superconductivity.

A note on samples: The internal ordering or mosaicity of the graphite crystalline regions inside commercial HOPG samples is given usually by the grade. For example, the highest ordered pyrolytic graphite samples have is a grade "A", which means a rocking curve width $\Delta \sim 0.4^{\circ} \pm 0.2^{\circ}$ ("B", $\Delta \sim 0.8^{\circ}$, etc.). Interestingly, and due to the contribution of two dimensional highly conducting internal interfaces between crystalline regions
$[7,10]$, the highest grade, i.e., smaller rocking curve width, does not always mean that the used sample provides the intrinsic transport properties of ideal graphite. The characterization of the internal structure of usual HOPG samples, as well as the thickness dependence of $R(T)$ to understand the transport and the magnetic properties of graphite, indicate that these two dimensional interfaces are of importance. The existence of rhombohedral inclusions $[19,20]$ (stacking order ABCABC instead of $\mathrm{ABAB}$ of the usual Bernal graphite structure) in HOPG as well as in Kish graphite samples can also have a relationship with the hidden superconductivity in graphite, following the theoretical work in Ref. [21]. According to literature (see e.g., Fig. 22 in Ref. [8]), the density of interfaces parallel to the graphene layers in Kish graphite, in regions of several microns length, is notable. Therefore, quantifying the perfection of any graphite sample through the resistivity ratio between $300 \mathrm{~K}$ and $4.2 \mathrm{~K}[8]$ is not necessarily the best criterion to be used if we are interested on the intrinsic properties of the graphene layers in graphite, because of the high conductivity of the interfaces in parallel to the graphene layers of the sample [10]. Two examples of the interfaces we are referring to can be seen in Fig. 1. On the other hand, commercial HOPG bulk samples are of high purity with average total impurity concentrations below $20 \mathrm{ppm}$. Especially the existence of magnetic impurities are of importance if the Defect-Induced Magnetism (DIM) is the main research issue. Their concentration remains below a few ppm for high grade HOPG samples [22].

The graphite flakes discussed in this work were obtained by exfoliation of HOPG samples of different batches, by careful mechanical press and rubbing the initial material on a previously cleaned substrate. As substrate, we used p-doped $\mathrm{Si}$ with a $150 \mathrm{~nm}$ SiN layer on top. We selected the flakes using microscopic and micro-Raman techniques to check their quality. More details on the preparation can be taken from Ref. [7] and other publications cited below.

This review is organized as follows. In the next section we discuss the experimental data for $R(T, H)$ from different graphite samples published in the last 12 years and argue that the first hints on unusual superconducting contribution can be already found in those measurements. In section III, we discuss the anomalous hysteresis in the magne- 
toresistance, a first clear indication for embedded granular superconductivity. Section IV, deals with the Josephson behavior measured in TEM lamellae whereas section $\mathrm{V}$ deals with the granular superconducting behavior found in the magnetization of water-treated graphite powder as well as in bulk HOPG samples for fields normal to the interfaces found inside those samples. In the last section, section VI, before the conclusion, we discuss possible origins for the superconducting signals on the basis of earlier and recent experimental and theoretical work.

\section{The behavior of the resistance vs. temperature at different applied magnetic fields}

In this section, we discuss the behavior of the resistance $R(T, H)$ of different HOPG samples including Kish graphite. The data we present here were taken from $[7,23,24]$ and a quick search in literature demonstrates that these data are reproducible and can be found in different publications, see, e.g., [2,25-27]. Figure 2(a) shows the $R(T)$ for different bulk graphite samples of different grades (rocking curve width) and for one sample (HOPG-1) at zero and under a magnetic field applied normal to the main area, i.e., normal to the graphene planes of the sample. This figure reveals a general behavior, namely that the lower the resistivity $\rho$ of the HOPG sample, the more metallic-like its temperature dependence. It is appealing to assume that these characteristics, low $\rho$, low $\Delta$ and the metallic behavior are clear signs for more ideal graphite. Therefore, from the measured $R(T)$, we may conclude that sample HOPG-3 is more ideal than sample HOPG-1 and the latter being more ideal than sample HOPG-2, see Fig. 2(a). This is indeed the usual interpretation found in several reviews in the literature, see e.g., [8,28].

From a quick look at all the curves in Fig. 2, however, one recognizes a striking similarity between them, although we are comparing different samples with different thickness and some of the curves were measured under a magnetic field applied normal to the graphene layers of the samples; also normal to the interfaces commonly found in some ordered samples $[7,8]$.

Let us start discussing the metallic-like behavior
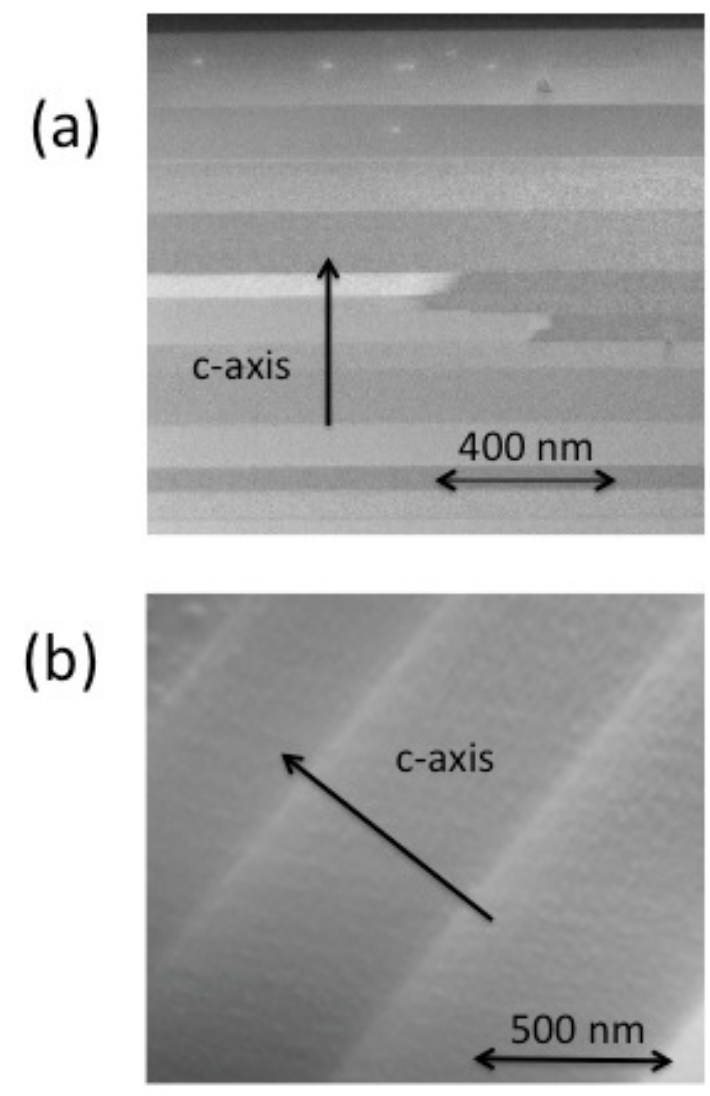

Figure 1: Transmission electron microscope pictures of two different kinds of interfaces and their distribution in HOPG samples. The TEM pictures were taken from two different lamellae, each about $300 \mathrm{~nm}$ thick and with the electron beam nearly parallel to the graphene planes of the samples. (a) The interfaces are recognized at the borders of crystalline regions of different gray colors. Taken from [7]. (b) Interfaces found in a HOPG sample used for magnetization measurements (see section V)that reveals hysteretic behavior in field and temperature. Taken from [29].

of $R(T)$ of sample HOPG-3 in Fig. 2(a). This sample behaves as the HOPG-UC sample shown in (c) at zero magnetic field, having also a maximum at $T \sim 150 \mathrm{~K}$. A "better" metallic character shows the HOPG sample in (b) or the Kish graphite sample in (d), without any maximum in the shown temperature range. Is this metallic-like behavior really intrinsic of ideal graphite? 
Papers in Physics, vol. 5, ART. 050007 (2013) / P. Esquinazi

The following experimental evidence does not support such interpretation:

First, for samples from the same batch, the metallic character of $R(T)$ vanishes in the whole temperature range when the sample thickness is below $\sim 50 \mathrm{~nm}[7,11,27]$, see Fig. 2(b).

Second, the metallic-like behavior vanishes in the whole temperature range after applying a magnetic field of the order of 1 to $2 \mathrm{kOe}$, an interesting behavior known as the Metal-Insulator Transition (MIT) [23, 25, 26], see Figs. 2(a), (c) and (d). Note that such magnetic field strength influences mainly the metallic-like region, see e.g., the change of sample HOPG-1 in Fig. 2(a) at zero and at $1 \mathrm{kOe}$ field, an interesting behavior noted first in [30] and interpreted as due to superconducting instabilities.

At those field strengths, i.e., $H \sim 1 \mathrm{kOe}$, the obtained $R(T)$ curves, for samples showing at zero field a metallic-like behavior, resemble the semiconducting-like curves obtained for sample HOPG-2 (Fig. 2(a)) or for samples with small thickness (Fig. 2(b)). At fields higher than a few $\mathrm{kOe}$, the rather large magnetoresistance of graphite starts to play the main role and the $R(T)$ curve increases in the whole temperature range.

Finally, all these results added to the existence of well defined interfaces in the metallic-like HOPG samples as well as in Kish graphite, with distances in the $c$-axis direction usually larger than $\sim 30 \mathrm{~nm}$, indicate that the metallic-like behavior is due to the contribution of these interfaces and it is not intrinsic of the graphene layers of ideal graphite $[7,10]$. Therefore, explanations of the MIT based on ideal graphite band models with a large number of free parameters $[25,26]$ are certainly not the appropriate ones.

All the different $R(T)$ curves for different samples shown in Fig. 2 and at zero field can be very well understood assuming the parallel contribution of semiconducting graphite paths in parallel to the one from the highly conducting interfaces [10]. The saturation of the resistance at $T \rightarrow 0 \mathrm{~K}$ is interpreted as due to the finite resistance of the sample surfaces (the free one and the one on the substrate) short circuiting the intrinsic behavior of the bulk graphene layers at low enough temperatures.

The question is now whether parts of these interfaces hide superconducting regions. It is certainly appealing to suggest that the huge MIT at fields normal to the interfaces (and below $\sim 2 \mathrm{kOe}$ ) is related to Josephson-coupled superconducting regions embedded in some of the interfaces. Note that the huge anisotropy of the MIT (parallel fields to the interfaces main plain do not affect the electrical transport) already implies that the regions responsible for the MIT must be laying parallel to the graphene layers [31]. Without the knowledge on the existence of these interfaces, an interpretation of the low field MIT based on the influence of superconductivity has been discussed in detail in the reviews $[24,32]$. In those reviews, one can recognize the remarkable similarity between the scaling approaches used to characterize the magnetic-fieldinduced superconductor-insulator quantum phase transition [33] or of the field-driven MIT in 2D electron (hole) systems [34] and the one obtained for the MIT observed in graphite. As we will see in the next sections, the experimental evidence obtained in the last recent years indicates that granular superconductivity exists within some of those interfaces, indeed.

If superconducting patches exist embedded in parts of the interfaces or in other two dimensional regions of the bulk ordered samples, one expects to measure some signs of granular superconductivity, as for example nonlinear $I-V$ curves or hysteresis in the magnetoresistance. However, this is not really observed in bulk large samples. There are at least two reasons for the apparent absence of these expected phenomena. One is the distribution of the input current between the ballistic channels given by the graphene layers $[17,18]$, the metallic, normal conducting parts of the interfaces and the regions where the superconducting patches exist. In other words, the usual maximum currents used in transport experiments reported in bulk samples may have been small enough so that the current through the superconducting regions remained below the critical Josephson one. The other reason is the experimental voltage sensitivity to measure the possible irreversibility in the magnetoresistance due to the existence of pinned vortices or fluxons. We will see in the next section that part of these problems can be overcome decreasing the sample size; in this way, one obtains the voltage signals from the regions of interest with enough sensitivity.

Apart from the large magnetic field sensitivity of the metallic-like resistance measure in bulk graphite samples with interfaces, is there any further hint for the existence of granular supercon- 
PAPERs in Physics, vol. 5, ART. 050007 (2013) / P. Esquinazi
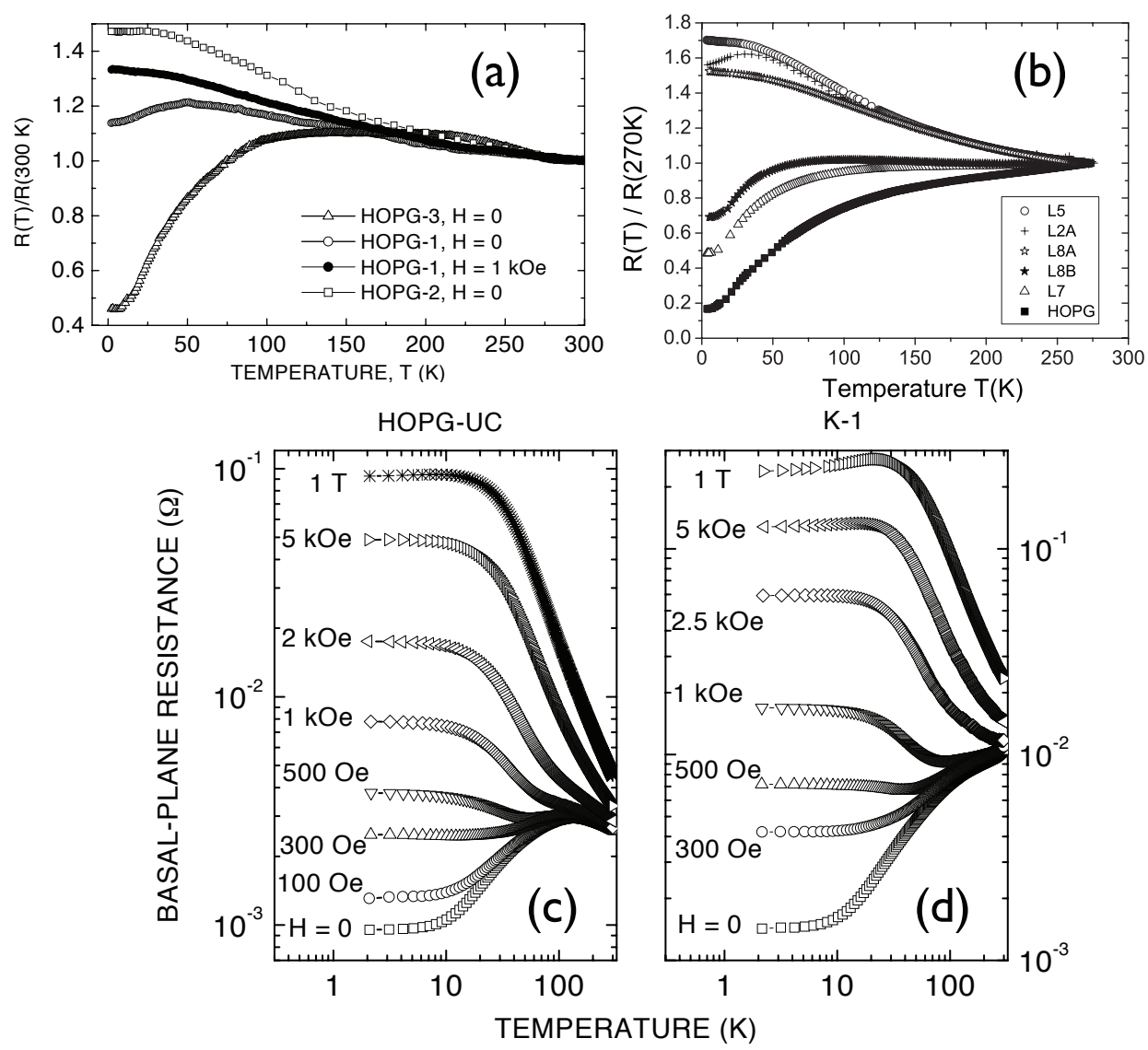

Figure 2: (a) Normalized resistance vs. temperature for three different HOPG bulk samples. The bottom, metallic-like curve corresponds to the sample HOPG-3, the curves above correspond to HOPG$1(H=0)$, HOPG-1 $(H=1 \mathrm{kOe})$, and HOPG-2. The grade and resistivity values are HOPG-1 $\left(\Delta=1.4^{\circ}\right.$, resistivity at $300 \mathrm{~K} \rho(300 \mathrm{~K})=45 \mu \Omega \mathrm{cm})$, HOPG-2 $\left(\Delta=1.2^{\circ}, \rho(300 \mathrm{~K})=135 \mu \Omega \mathrm{cm}\right)$ and HOPG-3 $\left(\Delta=0.5^{\circ}, \rho(300 \mathrm{~K})=5 \mu \Omega \mathrm{cm}\right)$. Taken from [23]. (b) Similar to (a) but for HOPG samples from the same batch but with different size, namely (thickness $\times$ length $\times$ width) L5: $12 \pm 3 \mathrm{~nm}, 27 \mu \mathrm{m}, 14 \mu \mathrm{m}$, L2A: $20 \pm 5 \mathrm{~nm}, 5 \mu \mathrm{m}, 10 \mu \mathrm{m}, \mathrm{L} 8 \mathrm{~A}: 13 \pm 2 \mathrm{~nm}, 14 \mu \mathrm{m}, 10 \mu \mathrm{m}, \mathrm{L} 8 \mathrm{~B}: 45 \pm 5 \mathrm{~nm}, 3 \mu \mathrm{m}, 3 \mu \mathrm{m}, \mathrm{L} 7$ : $75 \pm 5 \mathrm{~nm}, 17 \mu \mathrm{m}, 17 \mu \mathrm{m}$, HOPG: $17 \pm 2 \mu \mathrm{m}, 4.4 \mathrm{~mm}, 1.1 \mathrm{~mm}$, taken from [7]. (c) and (d) Resistance of bulk graphite samples vs. temperature at different applied fields normal to the graphene layers. The sample in (c) is a HOPG bulk sample from Union Carbide of grade A and the sample in (d) is Kish graphite. Taken from [24].

ductivity in those $R(T)$ curves? Yes, this hint is related to the thermally activated function $(\propto$ $\exp \left(-E_{a} / k_{B} T\right)$ with $E_{a}$, a sample dependent effective thermal barrier $\sim 30 \mathrm{~K}$ ) one needs in order to fit the metallic-like contribution below $T \sim 200 \mathrm{~K}$
[10]. This function is relevant in spite of only a factor five increase of the resistance between low and high temperatures, see Fig. 2. Skeptical readers can convince themselves about its relevance taking a similar example, as the exponential function 
Papers in Physics, vol. 5, ART. 050007 (2013) / P. Esquinazi

used to fit the increase, by a similar factor, of the ultrasonic attenuation with temperature below $T_{c}$ in conventional superconductors. We note that this exponential function has already been used to describe the increasing resistance of bulk graphite samples with temperature and it was speculated to be related to some superconducting-like behavior in graphite [32]. It is clear that this function is not the usual one, one expected for metals or semimetals and that cannot be understood within the usual electron-phonon interaction mechanisms, nor in two dimensions. A similar dependence has been observed in granular AlGe [35], which shows for a particular $\mathrm{Al}$ concentration a superconductor-semiconductor transition similar to that reported in Ref. [10] or, after an appropriate scaling in temperature, to some of the curves shown in Fig. 2. The observed thermally activated behavior might be understood on the basis of the Langer-Ambegaokar-McCumber-Halperin (LAMH) model $[36,37]$ that applies to narrow superconducting channels in which thermal fluctuations can cause phase slips. This interpretation gets further support from the evidence we discuss in the following sections.

\section{Hysteresis in the magnetoresis- tance}

In order to reveal by transport measurements the existence of granular superconductivity in some regions of the graphite samples, we need to increase the sensitivity of the measured voltage to those regions. To achieve this, we decrease the size of the sample enhancing in this way the probability to get some measurable influence of this phenomenon in the voltage. The work in Ref. [38] reported the first observations of an anomalous irreversible behavior in the magnetoresistance (MR) in a few tens of $\mathrm{nm}$ thick and several micrometer large multigraphene samples. Hysteresis in the magnetoresistance is a key evidence on the existence of either magnetic order (domains with their walls, for example) or vortices/fluxons and therefore on the existence of superconductivity. Because defects as well as hydrogen can trigger magnetic order in graphite, a first attempt would be to relate the measured hysteresis in the MR with the existence of magnetic order and magnetic domains, for example. How- ever, the data exhibited anomalous hysteresis loops in the MR [38], similar to those observed in granular superconductors with Josephson-coupled grains [39-41]. The anomalous hysteresis was observed only for magnetic fields perpendicular to the planes, whereas in the parallel to the planes direction, the MR remains negligible. This fact already points out to a remarkable large anisotropic response of the superconducting phase(s) in agreement with the hypothesis that these superconducting regions might be embedded in some of the interfaces found inside some bulk graphite samples $[7,8]$. The amplitude of the hysteresis in the MR reported in Ref. [38] vanishes at temperatures $T \sim 10 \mathrm{~K}$, clearly below the temperature at which the resistance shows a maximum, as it is the case for samples HOPG-1 in Fig. 2(a) or sample L2A in Fig. 2(b).

It is clear that thermal fluctuations can prevent the establishment of a coherent superconducting state in parts of the sample and therefore zero resistance state is not so simple to be achieved if the superconducting distribution is a mixture of superconducting patches at the interfaces and these are embedded in a multigraphene semiconducting matrix. Moreover, we should take also into account that the voltage electrodes are usually connected at the top surface of the graphite samples picking the voltage difference coming from a non-negligible normal conducting path.

One possibility to increase the sensitivity of the measured voltage to the field hysteresis these regions produce is to make a constriction in the middle of the two voltage electrodes, see inset in Fig. 3(a). In this case, we expect a locally narrower distribution of superconducting and normal regions at the constriction such that averaging effects should be less important. Simultaneously, through the constriction the main part of the voltage drop depends mostly on the region at the constriction, see Fig. 2(c) in Ref. [16]. Then, a higher sensitivity to the superconducting paths can be achieved in case they remain at or near the constriction. This idea has been successfully realized in [42] and its main results will be reviewed in this section.

Let us take two slightly different samples, 1 and 2 , with $R(T)$-curves as shown in Fig. 3(a). The aim of the experiment is to study the hysteresis in the MR those samples might show below the temperature at which a maximum in the resistance 

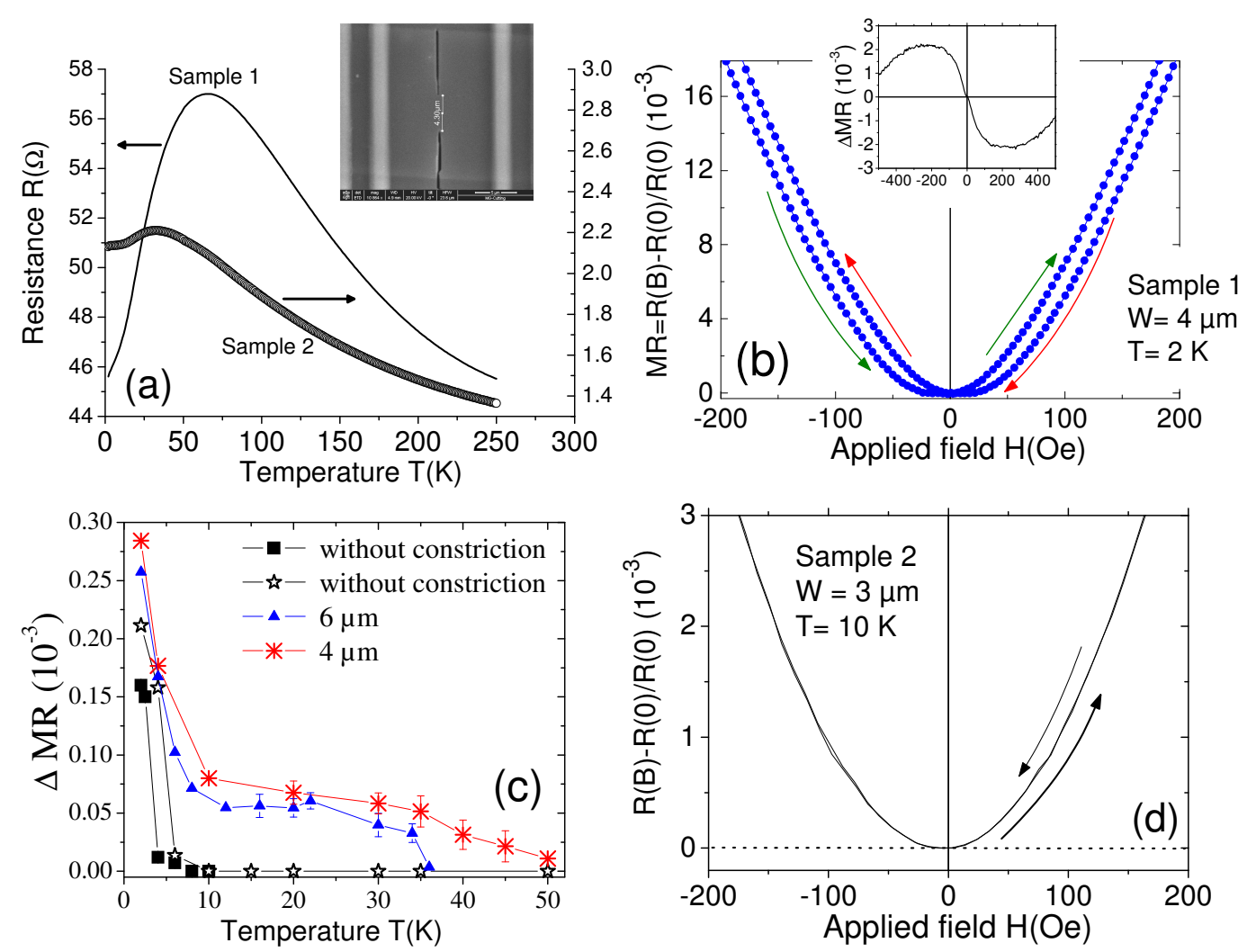

Figure 3: (a) Resistance vs. temperature, without constrictions and at zero applied field, for two graphite flakes of size (distance between voltage electrodes $\times$ width $\times$ thickness) for sample 1 (2): $13 \times 16 \times 0.015(2.6 \times 6 \times 0.040) \mu \mathrm{m}^{3}$. The observed temperature dependence remains for all constrictions widths. The inset shows a scanning electron microscope picture of sample 1 with a constriction width of $4.3 \mu \mathrm{m}$ between the two voltage electrodes. The scale bar is $5 \mu \mathrm{m}$. (b) Magnetoresistance (MR) vs. applied magnetic field for sample 1 with a $4 \mu \mathrm{m}$ constriction width and at $2 \mathrm{~K}$. The input current was $1 \mu \mathrm{A}$. Note the clear hysteresis in the MR when the field is swept from $\left|H_{\max }\right|=1000$ Oe. The inset shows the difference $\Delta \mathrm{MR}$ between the curve obtained starting from $H_{\max }=+1000$ Oe and the return curve measured from $H_{\min }=-1000$ Oe. (c) The absolute difference between the two MR curves of the hysteresis loop obtained at a fixed magnetic field of 16.6 Oe for sample 1 without constriction $(\star)$ and for two different constriction widths. The figure also shows the corresponding data for another graphite sample without constrictions ( $\mathbf{\square})$ from [38]. (d) Magnetoresistance measured from a starting maximum field of $1.4 \mathrm{kOe}$ at $10 \mathrm{~K}$ for sample 2 with a constriction width of $3 \mu \mathrm{m}$. Some of the figures and the data were taken from [42].

is measured, in case that maximum is related to the Josephson coupling between superconducting regions. Figure $3(\mathrm{~b})$ shows one example of the anomalous hysteresis loop in the MR. The going down curve (from high, positive to low, negative fields, red arrow), for example, runs below the go- ing up curve (green arrow) in the same quadrant as the field sweep was started, showing a minimum at positive fields of the order of 20 Oe, see also similar curves in Ref. [38]. To present the anomalous behavior clearly, the inset in Fig. 3(b) shows the difference between the two curves. This difference 
Papers in Physics, vol. 5, ART. 050007 (2013) / P. Esquinazi

is in clear contrast to the usual hysteresis in superconductors as well as ferromagnets [38,39], where the minimum (or maximum) in the MR is observed in the opposite field quadrant, and the increasing field resistance curve is usually below the decreasing field one.

Figure $3(\mathrm{c})$ shows the temperature dependence of the difference in the MR between the decreasing and increasing field curves at a fixed magnetic field for sample 1, without and with two constrictions. The results show that the smaller the constriction width, the higher the temperature at which the anomalous hysteresis is observed, decreasing below the sensitivity limit at $T>50 \mathrm{~K}$ for a constriction width of $4 \mu \mathrm{m}$, whereas the maximum in the $R(T)$ curve is at $\sim 70 \mathrm{~K}$, see Fig. 3(a). The absence of any hysteresis in the MR for sample 2 with a constriction width of $3 \mu \mathrm{m}$ and at $T=10 \mathrm{~K}$ indicates that the hysteresis does not come from some artifact due to the used focused ion beam method $[42,43]$ or due to an artifact in the measurement of the real field applied to the sample. As expected from the $R(T)$ curve, see Fig. 3(a), sample 2 shows the anomalous hysteresis in the MR at lower temperatures than sample 1 [42].

Summarizing this section, the observation of the anomalous hysteresis in the MR - together with the MIT and the relatively large MR at temperatures below the maximum in $R(T)$ - provides already striking hints that granular superconductivity is at work in some regions of these samples. The increase in the temperature region where the hysteresis is observed, decreasing the constriction width, demonstrates the problem of current averaging and voltage sensitivity limits usual experiments with large samples have.

\section{Direct evidence for Josephson behavior in the transport prop- erties of graphite: Measure- ments in TEM lamellae}

If the embedded interfaces (or some other quasi two dimensional regions) inside the measured graphite samples have superconducting properties, the best way to check them would be contacting electrodes as near as possible to those interfaces or interface regions and study the behavior as a function of any useful parameter one can take to influence their re- sponse. It should be clear that one cannot simply open the graphite sample at the interface and put voltage electrodes at the open surfaces of the interface, simply because it will not remain anymore. A tentative approach to put the contacts as near as possible to an interface has been done in Ref. [46]. Indeed, the observed behavior at low temperatures and as a function of magnetic field appeared to be superconducting-like.

A better and appealing evidence for the superconducting behavior embedded in some graphite ordered samples can be obtained by trying to locate the voltage electrodes directly at the inner edges of the interfaces. The work in Ref. [45] prepared TEM lamellae from bulk HOPG samples and using lithography and focused ion beam techniques, current and voltages electrodes at different positions of the samples were prepared. In this way, one tries to contact several of those interface edges simultaneously, as shown in Fig. 4(a). We note, however, that a thin surface layer of disordered graphite exists due to the $\mathrm{Ga}^{+}$ion irradiation used to cut the lamella from the bulk HOPG sample. This layer has a much larger resistance than the one of the graphene layers or of the interfaces [43] and therefore the input current goes through the lowest resistance path as well as the voltage electrodes pick up the response of the graphite sample with its interfaces. One can see this comparing first the $R(T)$ curves obtained at large enough currents in the lamellae (Fig. 4(c)) with those of graphite samples with top electrodes (Fig. 2). The fact that a zero resistance state (minimum voltage noise $\pm 5 \mathrm{nV}$ upon sample) is obtained at low currents with $I-V$ characteristic curves that resemble the one expects for Josephson coupled grains leaves little doubt about the origin of the obtained signals. In the TEM picture of Fig. 4(b), one can see the graphite single crystalline regions (different gray colors) oriented differently between them about the common $c$-axis and having well defined two dimensional interfaces, as high resolution TEM studies revealed [47].

Figure 4(c) shows the voltage vs. temperature measured in a TEM lamella of oriented graphite [45] at different input DC currents, from $100 \mathrm{nA}$ to $10 \mu \mathrm{A}$. The clear sharp transition, observed at $\sim 150 \mathrm{~K}$ at the lowest current, shifts to lower temperatures increasing the input DC current. For the largest input currents, the temperature dependence of the resistance of the contacted lamella shows a 
Papers in Physics, vol. 5, ART. 050007 (2013) / P. Esquinazi

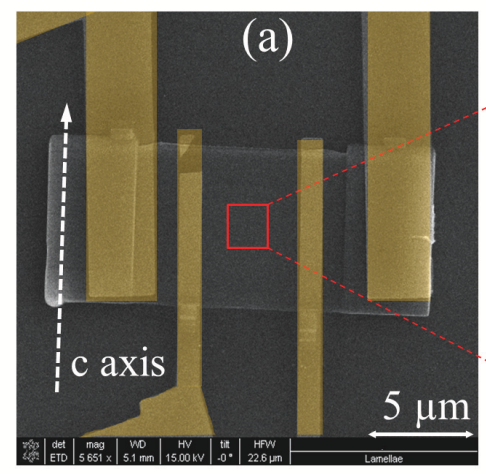

(b)
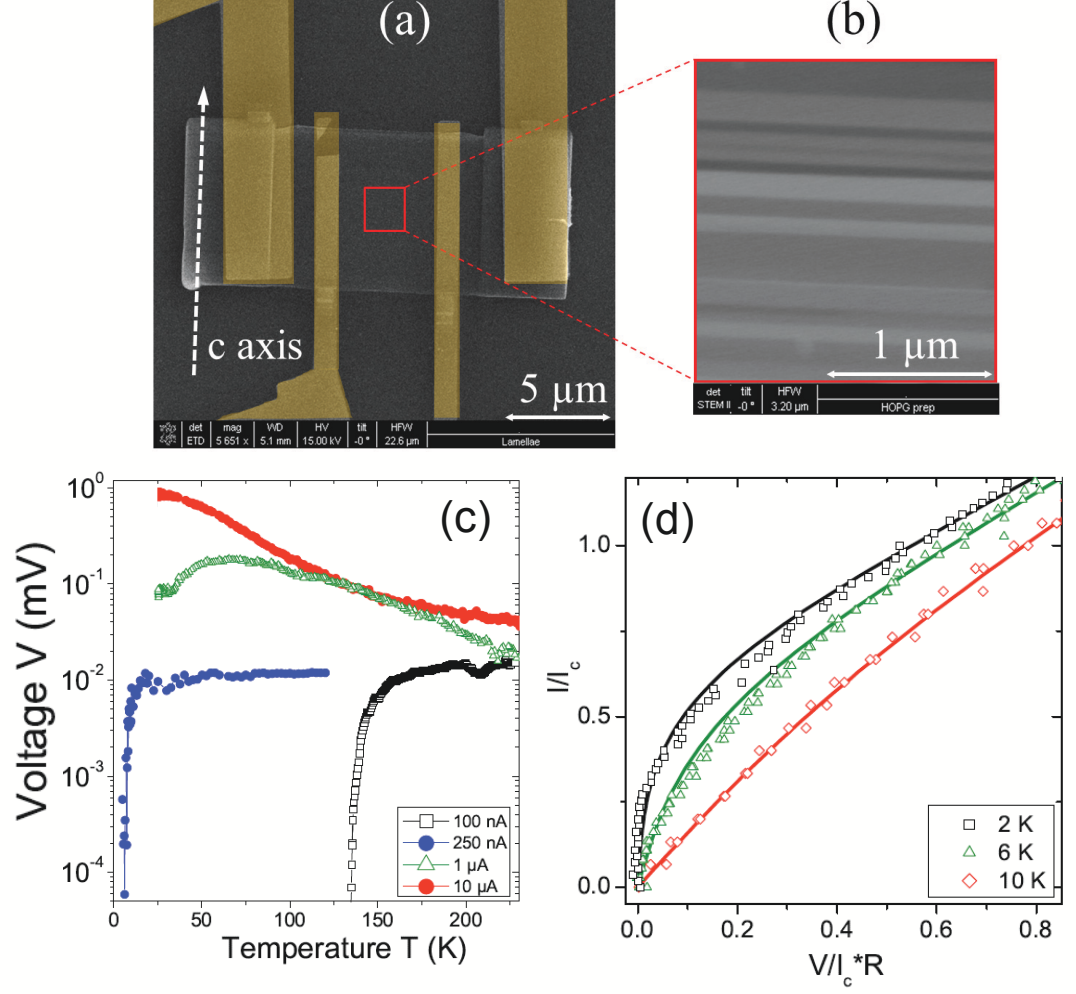

Figure 4: (a) Scanning Electron Microscopy (SEM) image of a lamella of $300 \mathrm{~nm}$ thickness on a $\mathrm{Si} / \mathrm{SiN}$ substrate where the yellowish colored areas are the electrodes. A four-point configuration has been prepared with the outer electrodes used to apply current and the inner ones to measure the voltage drop. The $c$-axis runs parallel to the substrate surface and normal to the current direction. (b) Transmission Electron Microscopy (TEM) image of a HOPG lamella. The different brightness corresponds to a different orientation within the $a-b$ plane of the crystalline regions with thickness $>30 \mathrm{~nm}$. (c) Voltage vs. temperature at different input currents for a lamella of $\sim 800 \mathrm{~nm}$ thickness and with Van der Pauw contact configuration. (d) Current-Voltage characteristics at different temperatures for a lamella of $\sim 300 \mathrm{~nm}$ thickness in reduced coordinates, where $R$ is the normal state resistance, $I$ the input current, and $I_{c}$ the critical Josephson current. The continuous curves are fitted to the model proposed in Ref. [44] with $I_{c}(T)$ as the only free parameter. Figures taken from [45].

maximum or follows the intrinsic semiconducting behavior of the graphene layers. This behavior already suggests the existence of high temperature granular superconductivity at some parts of the sample. The study reported in Ref. [45] shows that the transition temperature depends on the pre- pared sample. This indicates a sample dependent distribution of the superconducting regions and/or some influence of the preparation process or sample size on the superconductivity [47]. We also note that the observed sharp decrease in the measured voltage does not necessarily indicate the criti- 
Papers in Physics, vol. 5, ART. 050007 (2013) / P. Esquinazi

cal temperature of the superconducting regions but the temperature below which a percolative granular system shows negligible resistance due to the Josephson coupling at the used input current.

Current-voltage characteristic curves at different temperatures and in different lamellae obtained from different HOPG samples have been studied in Ref. [45]. An example of this $I-V$ curves at three temperatures is shown in Fig. 4(d) obtained for a different lamella. The curves follow the expected dependence for a Josephson junction [44] with a temperature dependent critical current, the only free parameter in the fit.

Further evidence that speaks for a superconducting origin of the $I-V$ curves is given by the expected detrimental effect of a magnetic field on the superconducting state. This effect can be due to an orbital depairing effect or due to the alignment of the electron spins at much higher fields, in case of singlet coupling. The effect of a magnetic field applied normal and parallel to the interfaces has been studied in detail for thick and thin lamellae in Ref. [45]. Upon sample size (thickness, i.e., width of the graphene planes inside the lamella) the observed effects are from the usual vanishing of the zero resistance state or no effect at all for thin lamellae. A magnetic field of a few $\mathrm{kOe}$ applied normal to the interfaces is enough to destroy the Josephson coupling at low temperatures, an effect compatible with the MIT observed in several graphite samples, see section II. Whereas a field applied parallel to them does not influence the $I-V$ curves at all, a fact that speaks for the two dimensionality of the superconducting regions.

Nevertheless, the influence of a magnetic field in HOPG samples with interfaces is not as "simple" as in conventional superconductors. For high fields applied normal to the interfaces, the $I-V$ curves show a recovery to the zero resistance state. The observed reentrance appears to be related to the magnetic-field driven reentrance observed at low temperatures in the longitudinal resistance at high enough magnetic fields [48]. This interesting behavior as well as the insensitivity of the $I-V$ curves to magnetic fields in very thin lamellae [45] deserve further studies.

We would like to note here that the possible effects of a magnetic field on the superconducting state of quasi two-dimensional superconductors, or in case the coupling does not correspond to a sin- glet state, are not that clear as in conventional superconductors. For example, results in two different two-dimensional superconductors, including one produced at the interfaces between non superconducting regions [49], show that superconductivity can even be enhanced by a parallel magnetic field. In case the pairing is $p$-type [50], the influence of a magnetic field is expected to be qualitatively different from the conventional, singlet coupling behavior $[51,52]$ with even an enhancement of the superconducting state at intermediate fields. In case the London penetration depth is much larger than the size of the superconducting regions at the interfaces of our lamellae or if the superconducting coherence length is of the order or larger than the thickness of the lamella, the influence of a magnetic field should be less detrimental.

Through these studies, and taking into account that in samples without these interfaces no signature of superconducting or metallic-like behavior has been observed (see also section V) it is appealing to suggest that superconductivity is somewhere hidden at some of those interfaces or interface regions. It should be also clear that not all those interfaces have superconducting regions with similar critical parameters. Those interfaces are formed during the preparation of the HOPG samples based on treatments at very high temperatures $\left(T>3400^{\circ} \mathrm{C}\right)$ and high pressures $\left(P \sim 10 \mathrm{~kg} / \mathrm{cm}^{3}\right)$ and in a non-systematic way [8]. Actually, they are not at all an aim of the production but actually the opposite, they should be avoided in order to enhance the crystal perfection of the bulk HOPG material. It is even possible that, upon the procedure used to control the structure and texture of the graphite sample, the near surface region, for example, can have a different degree of graphitization as inside the bulk HOPG sample [8]. This means that one may obtain different results from different parts of the same HOPG sample. Therefore, disconcerting situations and an apparent lack of reproducibility are preprogrammed in case the research studies are done without taking care of the internal microstructure of the studied samples.

\section{Magnetization measurements}

In this section, we present and discuss magnetization measurements done in bulk HOPG samples 
Papers in Physics, vol. 5, ART. 050007 (2013) / P. Esquinazi

with and without embedded interfaces and in water treated graphite powders. One of the main problems in interpreting magnetization data for fields applied parallel to the $c$-axis of the graphite structure, i.e., normal to the graphene layers and interfaces, is the need of subtraction of a large diamagnetic background. Due to the small amplitude of the superconducting-like signals in the studied samples, the subtraction of this linear in field background is not so simple, because it is not known with enough certainty to obtain the true field hysteresis after its subtraction. That means that we always have a certain arbitrariness in the shape of the obtained field hysteresis, a situation that will improve with the increase of the amount of material responsible for those superconducting-like signals. The small SQUID signals of interest imply that one should take additional efforts to minimize or rule out possible SQUID artifacts [53-55]. Therefore, systematic studies of samples of different or equal geometry and magnetic background, with and without interfaces, are necessary.

Taking into account: the overall shape of the hysteresis, the slope of the virgin curve at low fields where the subtraction does not affect too much, and the overall experience with ferromagnetic graphite $[22,56]$, one can rely to a certain extent on the obtained hysteresis shape. Certainly, not only the field hysteresis but also other evidence one gets from magnetization measurements as, e.g., the remanence at zero field as a function of the maximal field applied (see for example measurements for $\mathrm{YBa}_{2} \mathrm{Cu}_{3} \mathrm{O}_{7}$ in Ref. [57]) and the hysteresis in temperature dependent measurements helps to convince oneself about the existence of some kind of granular superconductivity. The hysteresis between the field cooled (FC) and zero-field cooled (ZFC) curves can help to discern between a superconducting or ferromagnetic-like behavior. The most obvious evidence that speaks against a simple ferromagnetic order of the hysteresis observed as a function of temperature and field is the two dimensionality of the obtained hysteretic signals [29], i.e., the superconducting-like signals are mainly measured for fields normal to the interfaces. This fact is not compatible with any kind of magnetic order including shape or magneto crystalline anisotropy, whatever large they might be. We note that the ferromagnetic response of graphite due to DIM is mostly measured for fields parallel to the graphene
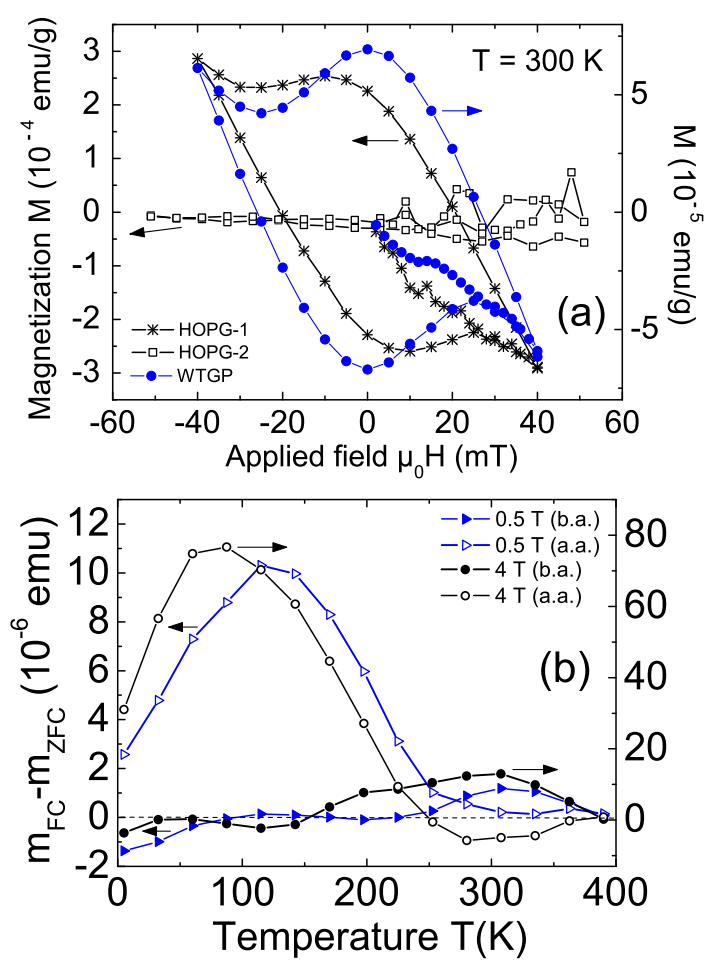

Figure 5: (a) Magnetization of two HOPG bulk samples (HOPG-1 and HOPG-2) after subtraction of a diamagnetic background and of water treated graphite powder (WTGP, right $y$-axis) at $300 \mathrm{~K}$. The HOPG-2 sample shows no hysteresis in contrast to the other two samples. (b) Temperature dependence of the difference between FC and ZFC magnetic moments of the HOPG-1 sample before (b.a.) and after (a.a.) warming the sample up to $\simeq 600 \mathrm{~K}$, at two constant applied fields, $0.5 \mathrm{~T}$ (left $y$-axis) and $4 \mathrm{~T}$ (right $y$-axis). The field was applied always normal to the interfaces or graphene planes of the samples. Data taken from [29].

layers, parallel to the main area of the samples [22].

\section{i. Bulk graphite samples}

Figure 5(a) shows the field hysteresis, after subtraction of the corresponding diamagnetic linear background, at $300 \mathrm{~K}$ of two bulk HOPG samples, HOPG-1 and HOPG-2 and a water treated graphite powder (WTGP) (right $y$-axis). A TEM characterization of the internal microstructure of 
the HOPG-1 sample shows clear evidence for well defined interfaces running parallel to the graphene layers, in contrast to the HOPG-2 sample [29], see Fig. 1(b). These results clearly indicate that the origin of the hysteresis is related to the existence of the interfaces in the HOPG-1 sample. The absence of the hysteresis in the HOPG-2 sample, which has a similar diamagnetic background and overall geometry as the HOPG-1 sample, also indicates that the hysteresis is not due to an obvious SQUID artifact or an artifact in the background subtraction. The field hysteresis is similar to that of WTGP. The narrowing of the hysteresis observed at high fields is expected for granular superconductors [58,60-62]. From the hysteresis, as well as measuring the remanent magnetic moment as a function of the applied field [29], one obtains the characteristic Josephson critical fields $h_{c 1}^{J}(T)$ and $h_{c 2}^{J}(T)$ with values similar to the WTGP [58] and a similar ratio $h_{c 2}^{J}(T) / h_{c 1}^{J}(T) \sim 3[29]$.

Figure 5(b) shows the magnetic moment hysteresis in temperature (FC minus the ZFC curve) for the HOPG-1 sample as received (b.a.) and after sweeping the temperature up to $500 \mathrm{~K}$ (a.a.) [29], at two applied fields. We would like to stress the following features: The hysteresis for the as-received sample starts from the turning point $(390 \mathrm{~K})$ and it is positive. The hysteresis in temperature at both applied fields are qualitatively similar, showing a crossing to negative values at low temperatures. Larger ZFC values (smaller in absolute value) than $\mathrm{FC}$ ones in the magnetic moment are usually not observed, neither in superconductors nor in ferromagnets and it does appear to be a SQUID artifact [29]. This negative hysteresis in temperature would suggest that the superconducting properties can be enhanced to some extent under a magnetic field, an effect that might be related to the reentrance we have shortly mentioned in section IV. A slight annealing of the HOPG-1 sample of less than one hour at $\sim 500 \mathrm{~K}$ changes drastically the observed hysteresis for both fields (open symbols in Fig. 5(b)). The hysteresis appears to be shifted to lower temperatures but with negative values at high temperatures and high fields. We note that annealing at similar temperatures for several hours produced a decrease in the overall hysteresis observed in WTGP (see supporting information of Ref. [58]). At the state of this research, it is unclear whether pinning properties of vortices and/or of fluxons or
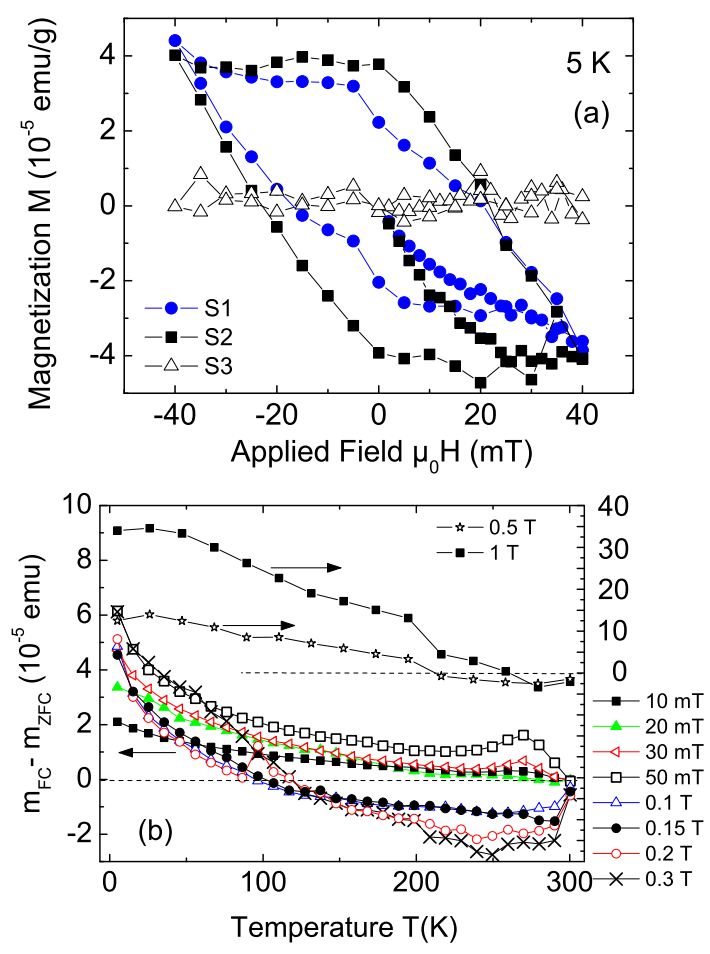

Figure 6: (a) Field hysteresis at $5 \mathrm{~K}$ for a maximum applied field of $40 \mathrm{mT}$ for the water treated graphite powder (S1), the same powder but after pressing it in a pellet with a pressure of $18 \pm 5 \mathrm{MPa}$ (S2) and after pressing it again with a pressure of $60 \pm 20 \mathrm{MPa}$ (S3). The corresponding diamagnetic linear backgrounds were subtracted from the measured data. (b) Difference between the FC and ZFC curve at different applied fields for a water treated graphite powder. Data taken from Ref. [58].

the existence of different superconducting phases play a main role in the hysteresis that is observed for fields applied normal to the interfaces.

\section{ii. Water treated graphite powder}

The work of Ref. [58] reports on the magnetic response of WTGPs. The main message of that work is that the WTGP shows a hysteretic behavior in field and temperature compatible with granular superconductivity. As an example, we show in Fig. 6(a) the field hysteresis at $5 \mathrm{~K}$ of a WTGP (S1, lose powder without applying significant pres- 
Papers in Physics, vol. 5, ART. 050007 (2013) / P. Esquinazi

sure) and the same WTGP but after pressing it into a pellet with two different pressures (S2,S3). After the diamagnetic background subtraction, the field hysteresis is similar to that obtained for bulk HOPG sample with interfaces, see Fig. 5(a) for similar data but at $300 \mathrm{~K}$. The fact that the hysteresis vanishes after applying pressure to the powder rules out simple SQUID artifacts (the diamagnet background does not diminish after making a pellet from the graphite powder, but the contrary) and also it rules out that the hysteresis is due to a ferromagnetic response due to impurities.

Figure 6(b) shows the difference in the magnetic moment between the ZFC and FC curves, as in Fig. 5(b). The behavior of this difference as a function of the applied field appears to be compatible with the one expected for granular superconductors [58]. Note the following features: The hysteresis increases at all $T$ for fields $\mu_{0} H \lesssim 50 \mathrm{mT}$, showing a maximum near the turning point of $300 \mathrm{~K}$, similar to the HOPG-1 sample in the as-received state, see Fig. 5(b). At fields $0.1 \mathrm{~T} \lesssim \mu_{0} H \lesssim 0.2 \mathrm{~T}$ the difference decreases at all $T$ and remains rather field independent. At higher fields, however, it increases showing a shift of the crossing point (from negative to positive values) to higher $T$. This behavior is at odds to the one expected for ferromagnets, even for ferromagnetic nanoparticles [63] as well as for superconductors with a pinning force that decreases with applied field in the shown field range. From the results in [58], and using basic concepts of vortex pinning, we would then conclude that if an upper critical field exists, then it should be clearly larger than $7 \mathrm{~T}$ in the temperature range of the figure.

In spite of some interesting differences between the behavior obtained for bulk HOPG and WTGP, the similarities already suggest that the water treatment helps to produce a certain amount of interfaces between graphite grains, being the origin for the whole hysteresis. Thermal annealing as well as pressing the WTGP are detrimental indicating that defects and/or hydrogen or oxygen at the interfaces could play an important role in the observed phenomena.

\section{Discussion}

Superconductivity in carbon-based systems is a rather old, well recognized fact. This phenomenon was probably first observed in the potassium intercalated graphite $C_{8} K[64]$ back in 1965. Since then, a considerable amount of studies reported this phenomenon in carbon-based systems, reaching critical temperatures $T_{c} \sim 10 \mathrm{~K}$ in intercalated graphite $[65,66]$ and above $30 \mathrm{~K}$ - though not percolative - in some HOPG samples [59] as well as in doped graphite and amorphous carbon systems [67-70]. Traces of superconductivity at $T_{c}=65 \mathrm{~K}$ have been recently reported in amorphous carbon powder that contained a small amount of sulfur [71]. Superconductivity was found also in carbon nanotubes with $T_{c}=0.55 \mathrm{~K}[72]$ and $12 \mathrm{~K}[73]$ or possibly even higher critical temperatures $[74,75]$. Superconductivity with $T_{c} \sim 4 \mathrm{~K}$ in boron-doped diamond [76] and in diamond films with $T_{c} \sim 7 \mathrm{~K}$ [77] belong also to the recently published list of carbon-based superconductors. We should note, however, that superconductivity at room temperature in a disordered graphite powder has been already reported in 1974 [78], see also [79], a work that did not attract the necessary attention in the community.

Whether quasi two dimensional interfaces play a role in the above mentioned carbon-based superconductors, one can probably rule out only for the intercalated graphite and doped diamond compounds, where the three dimensional superconductivity is characterized by a relatively low critical temperature. We may speculate that the traces of superconductivity found in doped amorphous carbon, disordered or ordered graphite powders may be related to some interfaces between well ordered graphite regions. The experience of the high temperature superconducting oxides already suggests that two dimensionality is advantageous to achieve higher critical temperatures.

Apart from the usual transport and magnetization measurements used to characterize the superconducting state, there are scanning tunneling spectroscopy (STS) results obtained on certain disordered regions of a HOPG surface at $T=4.2 \mathrm{~K}$ that revealed an apparent energy gap $\sim 100 \mathrm{meV}[80]$. Although the overall curves resemble a superconducting-like density of states, the authors suggested that the gap originates from charging effects. See further STS results and the discus- 
PAPers in Physics, vol. 5, ART. 050007 (2013) / P. Esquinazi

sion in [70].

Theoretical works that deal with superconductivity in graphite as well as in graphene have been published in recent years. For example, $p$-type superconductivity has been predicted to occur in inhomogeneous regions of the graphite structure [50] or $d$-wave high- $T_{c}$ superconductivity [81] based also on resonance valence bonds [82], or at the graphite surface region with rhombohedral stacking due to a topologically protected flat band [83].

For the graphite structure, the experimental evidence obtained in the last years suggests that high temperature superconductivity exists at certain interfaces or interface regions within the usual Bernal structure although the structure of the superconducting regions remains unknown. One can further speculate that due to the high carrier concentration that can be localized at those interfaces, they should be predestined to play a role in triggering superconductivity. Following a BCS approach in two dimensions (with anisotropy), for example, a critical temperature $T_{c} \sim 60 \mathrm{~K}$ has been estimated if the density of conduction electrons per graphene plane increases to $n \sim 10^{14} \mathrm{~cm}^{-2}$, a density that might be induced by defects and/or hydrogen adatoms [84] at the interfaces, or by $\mathrm{Li}$ deposition [85]. Further predictions for superconductivity in graphene support the premise that $n>10^{13} \mathrm{~cm}^{-2}$ in order to reach $T_{c}>1 \mathrm{~K}[86,87]$. On the other hand, the possibility to have high temperature superconductivity at the surface of or in the rhombohedral graphite phase $[21,83]$ - a phase that sometimes is found in graphite samples $[19,20]$ - stimulates further careful studies of these hidden interfaces. In the last years, superconductivity has been found at the interfaces between oxide insulators [88] as well as between metallic and insulating copper oxides with $T_{c} \gtrsim 50 \mathrm{~K}[89]$. Also, interfaces in different $\mathrm{Bi}$ bicrystals show superconductivity up to $21 \mathrm{~K}$, although Bi bulk is not a superconductor [90, 91].

Finally, we think that some of the interfaces are also the origin for the metallic-like behavior of graphite samples as well as for the quantum Hall effect (QHE) found in some HOPG samples [48,92]. Because the existence, density as well as the intrinsic properties of these interfaces depend on sample, we can now understand why the reproducibility of the QHE in bulk HOPG samples is rather poor.

\section{Conclusion}

In this review, we have discussed the following experimental evidence:

Firstly, the temperature and magnetic field dependence of the electrical resistance of bulk and thin films of graphite samples and its relation with the existence of two dimensional interfaces.

Secondly, the Josephson behavior of the currentvoltage curves with an apparent zero resistance state at high temperatures in especially made TEM lamellae.

Thirdly, the anomalous hysteresis in the magnetoresistance observed in graphite thin samples as well as its enhancement restricting the current path within the sample.

Finally, the overall magnetization of bulk graphite samples, with and without interfaces, as well as water treated graphite powders.

All this experimental evidence as a whole indicates the existence of superconductivity located at certain interfaces inside graphite samples. Although we cannot rule out other interpretations for some of the observations discussed in this work, the whole evidence suggests that superconductivity should be the origin for all the phenomena discussed here. Clearly, the situation is still highly unsatisfactory because several open questions remain, namely, the characteristics of the superconducting phase(s), from the structure to the main superconducting parameters, as "simple" as the critical temperature and critical fields, the coherence and penetration lengths, etc. It is clear that further studies are necessary in the future but the overall work done until now shows us the way to go.

Acknowledgements - The author acknowledges the support provided by the Deutsche Forschungsgemeinschaft under contract DFG ES 86/16-1 and the ESF-Nano under the Graduate School of Natural Sciences "BuildMona". The results presented in this review were part of the Ph.D. thesis of Heiko Kempa (section II), Srujana Dusari (section III) and Ana Ballestar (section IV) as well as the master thesis of Thomas Scheike (section V) done in the Division of Superconductivity and Magnetism of the Institute for Experimental Physics II of the University of Leipzig. The author thanks Dipl. 
Papers in Physics, vol. 5, ART. 050007 (2013) / P. Esquinazi

Kris. Annette Setzer, Dr. José Barzola-Quiquia and Dr. Winfried Böhlmann for their experimental assistance and support. The permanent support as well as the discussions with Nicolás García are gratefully acknowledged. Special thanks go to Yakov Kopelevich with whom we started in the year 1999 and in a rather naive way the research of a new and unexpected world behind graphite.

\section{Note added in proof}

Since the submission of this manuscript, some new works related to the subject of this review were published. Tight-binding simulations done in Ref. [93] support the work done in Ref. [21] and found that surface superconductivity is robust for $\mathrm{ABC}$ stacked multilayer graphene, even at very low pairing potentials. Through the observation of persistent currents in a graphite filled ring-shaped container immersed in alkanes, the author in Ref. [94] claimed possible room temperature superconductivity. For completeness, we include them in the reference list.

[1] J W McClure, Energy band structure of graphite, IBM J. Res. Dev. 8, 255 (1964).

[2] B T Kelly, Physics of graphite, Applied Science Publishers, London (1981).

[3] A Grüneis, C Attaccalite, T Pichler, V Zabolotnyy, $\mathrm{H}$ Shiozawa, $\mathrm{S}$ L Molodtsov, D Inosov, A Koitzsch, M Knupfer, J Schiessling, R Follath, R Weber, P Rudolf, R Wirtz, A Rubio, Electron-electron correlation in graphite: A combined angle-resolved photoemission and first-principles study, Phys. Rev. Lett. 100, 037601 (2008).

[4] I M Tsidilkovski, Electron spectrum of gapless semiconductors. Springer Series in Solid-State Sciences Vol. 116, Springer Verlag (1997).

[5] R O Dillon, I L Spain, J W McClure, Electronic energy band parameters of graphite and their dependence on pressure, temperature and acceptor concentration, J. Phys. Chem. Solids 38, 635 (1977).
[6] J M Schneider, M Orlita, M Potemski, D K Maude, Consistent interpretation of the lowtemperature magnetotransport in graphite using the Slonczewski-Weiss-McClure 3D bandstructure calculations, Phys. Rev. Lett. 102, 166403 (2009).

[7] J Barzola-Quiquia, J L Yao, P Rödiger, $\mathrm{K}$ Schindler, P Esquinazi, Sample size effects on the transport properties of mesoscopic graphite samples, Physica Status Solidi A 205, 2924 (2008).

[8] M Inagaki, New carbons: Control of structure and functions, Elsevier (2000).

[9] A Arndt, D Spoddig, P Esquinazi, J BarzolaQuiquia, S Dusari, T Butz, Electric carrier concentration in graphite: Dependence of electrical resistivity and magnetoresistance on defect concentration, Phys. Rev. B 80, 195402 (2009).

[10] N García, P Esquinazi, J Barzola-Quiquia, S Dusari, Evidence for semiconducting behavior with a narrow band gap of Bernal graphite, New J. Phys. 14, 053015 (2012).

[11] Y Ohashi, K Yamamoto, T Kubo, Shubnikov - de Haas effect of very thin graphite crystals, In: Carbon'01, An International Conference on Carbon, Pag. 568, The American Carbon Society, Lexington, KY, United States, (2001).

[12] B C Camargo, Y Kopelevich, S B Hubbard, A Usher, W Böhlmann, P Esquinazi, Effect of structural disorder on the quantum oscillations in graphite (unpublished). In this work the authors show that in certain HOPG samples (SPI) of high grade, the density of interfaces is much lower than in, for example, Advanced Ceramics HOPG ZYA samples. In this new HOPG samples basically no SdH oscillations are found and the temperature dependence of the resistance shows a semiconducting behavior with saturation a low temperatures (2013).

[13] M Orlita, C Faugeras, G Martinez, D K Maude, M L Sadowski, J M Schneider, M Potemski, Magneto-transmission as a probe of Dirac fermions in bulk graphite, J. Phys: Cond. Mat. 20, 454223 (2008). 
Papers in Physics, vol. 5, ART. 050007 (2013) / P. Esquinazi

[14] N A Goncharuk, L Nádvorník, C Faugeras, M Orlita, L Smrčka, Infrared magnetospectroscopy of graphite in tilted fields, Phys. Rev. B 86, 155409 (2012).

[15] J C González, M Muñoz, N García, J BarzolaQuiquia, D Spoddig, K Schindler, P Esquinazi, Sample-size effects in the magnetoresistance of graphite, Phys. Rev. Lett. 99, 216601 (2007).

[16] N García, P Esquinazi, J Barzola-Quiquia, B Ming, D Spoddig, Transition from ohmic to ballistic transport in oriented graphite: Measurements and numerical simulations, Phys. Rev. B 78, 035413 (2008).

[17] S Dusari, J Barzola-Quiquia, P Esquinazi, N García, Ballistic transport at room temperature in micrometer-size graphite flakes, Phys. Rev. B 83, 125402 (2011).

[18] P Esquinazi, J Barzola-Quiquia, S Dusari, N García, Length dependence of the resistance in graphite: Influence of ballistic transport, J. Appl. Phys. 111, 033709 (2012).

[19] Q Lin, T Li, Z Liu, Y Song, L He, Z Hu, Q Guo, H Ye, High-resolution TEM observations of isolated rhombohedral crystallites in graphite blocks, Carbon 50, 2369 (2012).

[20] C H Lui, Z Li, Z Chen, P V Klimov, L E Brus, $\mathrm{T}$ F Heinz, Imaging stacking order in few-layer graphene, Nano Lett. 11, 164 (2011).

[21] N B Kopnin, M Ijäs, A Harju, T T Heikkilä, High-temperature surface superconductivity in rhombohedral graphite, Phys. Rev. B 87, 140503 (2013).

[22] P Esquinazi, J Barzola-Quiquia, D Spemann, M Rothermel, H Ohldag, N García, A Setzer, $\mathrm{T}$ Butz, Magnetic order in graphite: Experimental evidence, intrinsic and extrinsic difficulties, J. Magn. Magn. Mat. 322, 1156 (2010).

[23] H Kempa, Y Kopelevich, F Mrowka, A Setzer, J H S Torres, R Höhne, P Esquinazi, Magnetic field driven superconductor-insulatortype transition in graphite, Solid State Commun. 115, 539 (2000).
[24] Y Kopelevich, P Esquinazi, J H S Torres, R R da Silva, H Kempa, Graphite as a highly correlated electron liquid, In: Advances in Solid State Physics, Vol. 43, Ed. B Kramer, Pag. 207, Springer-Verlag, Berlin (2003).

[25] T Tokumoto, E Jobiliong, E Choi, Y Oshima, J Brooks, Electric and thermoelectric transport probes of metal-insulator and two-band magnetotransport behavior in graphite, Solid State Commun. 129, 599 (2004).

[26] X Du, S W Tsai, D L Maslov, A F Hebard, Metal-insulator-like behavior in semimetallic bismuth and graphite, Phys. Rev. Lett. 94, 166601 (2005).

[27] Y Zhang, J P Small, W V Pontius, P Kim, Fabrication and electric-field-dependent transport measurements of mesoscopic graphite devices, Appl. Phys. Lett. 86, 073104 (2005).

[28] See several reviews in: Graphite and precursors, World of carbon series, Vol. 1, Ed. P Delhaes, Gordon and Breach Science Publishers (2001).

[29] T Scheike, P Esquinazi, A Setzer, W Böhlmann, Granular superconductivity at room temperature in bulk highly oriented pyrolytic graphite samples, Carbon 59, 140 (2013).

[30] Y Kopelevich, V Lemanov, S Moehlecke, J Torres, Landau level quantization and possible superconducting instabilities in highly oriented pyrolitic graphite, Phys. Solid State 41, 1959 (1999).

[31] H Kempa, H C Semmelhack, P Esquinazi, $\mathrm{Y}$ Kopelevich, Absence of metal-insulator transition and coherent interlayer transport in oriented graphite in parallel magnetic fields, Solid State Commun. 125, 1 (2003).

[32] Y Kopelevich, P Esquinazi, J Torres, R da Silva, H Kempa, F Mrowka, R Ocaña, Metal-insulator-metal transitions, superconductivity and magnetism in graphite, In: Studies of High Temperature Superconductors, Vol. 45, Chap. 3, Pag. 59, Nova Science Publishers Inc. (2003). 
Papers in Physics, vol. 5, ART. 050007 (2013) / P. Esquinazi

[33] M P A Fisher, Quantum phase transitions in disordered two-dimensional superconductors, Phys. Rev. Lett. 65, 923 (2000).

[34] E Abrahams, S V Kravchenko, M P Sarachik, Metallic behavior and related phenomena in two dimensions, Rev. Mod. Phys. 73, 251 (2001).

[35] Y Shapira, G Deutscher, Semiconductorsuperconductor transition in granular Al-Ge, Phys. Rev. B 27, 4463 (1983).

[36] J S Langer, V Ambegaokar, Intrinsic resistive transition in narrow superconducting channels, Phys. Rev. 164, 498 (1967).

[37] D E McCumber, B I Halperin, Time scale of intrinsic resistive fluctuations in thin superconducting wires, Phys. Rev. B 1, 1054 (1970).

[38] P Esquinazi, N García, J Barzola-Quiquia, $\mathrm{P}$ Rödiger, K Schindler, J L Yao, M Ziese, Indications for intrinsic superconductivity in highly oriented pyrolytic graphite, Phys. Rev. B 78, 134516 (2008).

[39] L Ji, M S Rzchowski, N Anand, M Thinkam, Magnetic-field-dependent surface resistance and two-level critical-state model for granular superconductors, Phys. Rev. B 47, 470 (1993).

[40] Y Kopelevich, C dos Santos, S Moehlecke, A Machado, Current-induced superconductorinsulator transition in granular high- $T_{c}$ superconductors, arXiv:0108311 (2001).

[41] I Felner, E Galstyan, B Lorenz, D Cao, Y S Wang, Y Y Xue, C W Chu, Magnetoresistance hysteresis and critical current density in granular $\mathrm{RuSr}_{2} \mathrm{Gd}_{2-x} \mathrm{Ce}_{x} \mathrm{Cu}_{2} \mathrm{O}_{10-\delta}$, Phys. Rev. B 67, 134506 (2003).

[42] S Dusari, J Barzola-Quiquia, P Esquinazi, Superconducting behavior of interfaces in graphite: Transport measurements of microconstrictions, J. Supercond. Nov. Magn. 24, 401 (2011).

[43] J Barzola-Quiquia, S Dusari, G Bridoux, F Bern, A Molle, P Esquinazi, The influence of $\mathrm{Ga}^{+}$irradiation on the transport properties of mesoscopic conducting thin films, Nanotech. 21, 145306 (2010).
[44] V Ambegaokar, B I Halperin, Voltage due to thermal noise in the DC Josephson effect, Phys. Rev. Lett. 22, 1364 (1969).

[45] A Ballestar, J Barzola-Quiquia, T Scheike, $\mathrm{P}$ Esquinazi, Evidence of Josephson-coupled superconducting regions at the interfaces of highly oriented pyrolytic graphite, New J. Phys. 15, 023024 (2013).

[46] J Barzola-Quiquia, P Esquinazi, Ferromagnetic- and superconducting-like behavior of the electrical resistance of an inhomogeneous graphite flake, J. Supercond. Nov. Magn. 23, 451 (2010).

[47] A Ballestar, P Esquinazi, Highly oriented pyrolytic graphite TEM lamellae preparation to study transport properties of the internal interfaces, J. Visual. Exp. (in press).

[48] Y Kopelevich, J H S Torres, R R da Silva, F Mrowka, H Kempa, P Esquinazi, Reentrant metallic behavior of graphite in the quantum limit, Phys. Rev. Lett. 90, 156402 (2003).

[49] H J Gardner, A Kumar, L Yu, P Xiong, M P Warusawithana, L Wang, O Vafek, D G Schlom, Enhancement of superconductivity by a parallel magnetic field in two-dimensional superconductors, Nat. Phys. 7, 895 (2011).

[50] J González, F Guinea, M A H Vozmediano, Electron-electron interactions in graphene sheets, Phys. Rev. B 63, 134421 (2001).

[51] K Scharnberg, R A Klemm, p-wave superconductors in magnetic fields, Phys. Rev. B 22, 5233 (1980).

[52] A Knigavko, B Rosenstein, Spontaneous vortex state and ferromagnetic behavior of typeII p-wave superconductors, Phys. Rev. B 58, 9354 (1998).

[53] N Casan-Pastor, P Gomez-Romero, L C Baker, Magnetic measurements with a squid magnetometer: Possible artifacts induced by sample holder off centering, J. Appl. Phys. 69, 5088 (1991).

[54] A Ney, T Kammermeier, V Ney, K Ollefs, $\mathrm{S}$ Ye, Limitations of measuring small magnetic 
Papers in Physics, vol. 5, ART. 050007 (2013) / P. Esquinazi

signals of samples deposited on a diamagnetic substrate, J. Magn. Magn. Mater. 320, 3341 (2008).

[55] M Sawicki, W Stefanowicz, A Ney, Sensitive SQUID magnetometry for studying nanomagnetism, Semicond. Sci. Tech. 26, 064006 (2011).

[56] J Barzola-Quiquia, W Böhlmann, P Esquinazi, A Schadewitz, A Ballestar, S Dusari, L Schultze-Nobre, B Kersting, Enhancement of the ferromagnetic order of graphite after sulphuric acid treatment, Appl. Phys. Lett. 98, 192511 (2011).

[57] M W McElfresh, Y Yeshurun, A P Malozemoff, F Holtzberg, Remanent magnetization, lower critical fields and surface barriers in an $\mathrm{YBa}_{2} \mathrm{Cu}_{3} \mathrm{O}_{7}$ crystal, Physica A 168, 308 (1990).

[58] T Scheike, W Böhlmann, P Esquinazi, J Barzola-Quiquia, A Ballestar, A Setzer, Can doping graphite trigger room temperature superconductivity? Evidence for granular hightemperature superconductivity in water-treated graphite powder, Adv. Mater. 24, 5826 (2012).

[59] Y Kopelevich, P Esquinazi, J Torres, S Moehlecke, Ferromagnetic- and superconducting-like behavior of graphite, J. Low Temp. Phys. 119, 691 (2000).

[60] S Senoussi, C Aguillon, S Hadjoudj, The contribution of the intergrain currents to the low field hysteresis cycle of granular superconductors and the connection with the micro- and macrostructures, Physica C 175, 215 (1991).

[61] M Borik, M Chernikov, V Veselago, $\mathrm{V}$ Stepankin, Anomalies of the magnetic properties of granular oxide superconductor $\mathrm{BaPb}_{l-x} \mathrm{Bi}_{x} \mathrm{O}_{3}$, J. Low Temp. Phys. 85, 283 (1991).

[62] B Andrzejewski, E Guilmeau, C Simon, Modelling of the magnetic behaviour of random granular superconductors by the single junction model, Supercond. Sci. Tech. 14, 904 (2001).
[63] R Prozorov, Y Yeshurun, T Prozorov, A Gedanken, Magnetic irreversibility and relaxation in assembly of ferromagnetic nanoparticles, Phys. Rev. B 59, 6956 (1999).

[64] N B Hannay, T H Geballe, B T Matthias, K Andres, P Schmidt, D MacNair, Superconductivity in graphitic compounds, Phys. Rev. Lett. 14, 225 (1965).

[65] T E Weller, M Ellerby, S S Siddharth, R P Smith, T Skippe, Superconductivity in the intercaled graphite compounds $C_{6} \mathrm{Yb}$ and $C_{6} \mathrm{Ca}$, Nat. Phys. 1, 39 (2005).

[66] N Emery, C Hérold, M D’Astuto, V Garcia, C Bellin, J F Marêché, P Lagrange, G Loupias, Superconductivity of bulk $\mathrm{CaC}_{6}$, Phys. Rev. Lett. 95, 035413 (2005).

[67] R R da Silva, J H S Torres, Y Kopelevich, Indication of superconductivity at $35 \mathrm{~K}$ in graphitesulfur composites, Phys. Rev. Lett. 87, 147001 (2001).

[68] Y Kopelevich, R R da Silva, J H S Torres, S Moehlecke, M B Maple, High-temperature local superconductivity in graphite-sulfur composites, Physica C 408, 77 (2004).

[69] I Felner, Y Kopelevich, Magnetization measurement of a possible high-temperature superconducting state in amorphous carbon doped with sulfur, Phys. Rev. B 79, 233409 (2009).

[70] Y Kopelevich, P Esquinazi, Ferromagnetism and superconductivity in carbon-based systems, J. Low Temp. Phys. 146, 629 (2007).

[71] I Felner, O Wolf, O Millo, High-temperature superconductivity in sulfur-doped amorphous carbon systems, J. Supercond. Nov. Magn. 25, 7 (2012).

[72] M Kociak, A Y Kasumov, S Guéron, B Reulet, I I Khodos, Y B Gorbatov, V T Volkov, L Vaccarini, H Bouchiat, Superconductivity in ropes of single-walled carbon nanotubes, Phys. Rev. Lett. 86, 2416 (2001).

[73] I Takesue, J Haruyama, N Kobayashi, S Chiashi, S Maruyama, T Sugai, H Shinohara, Superconductivity in entirely end-bonded multiwalled carbon nanotubes, Phys. Rev. Lett. 96, 057001 (2006). 
Papers in Physics, vol. 5, ART. 050007 (2013) / P. Esquinazi

[74] Z K Tang, L Zhang, N Wang, X X Zhang, G H Wen, G D Li, J N Wang, C T Chan, P Sheng, Superconductivity in 4 angstrom single-walled carbon nanotubes, Science 292, 2462 (2001).

[75] G M Zhao, Y S Wang, Possible superconductivity above $400 k$ in carbon-based multiwall nanotubes, arXiv:0111268 (2001).

[76] E A Ekimov, V A Sidorov, E D Bauer, N N Mel'nik, N J Curro, J D Thompson, S M Stishov, Superconductivity in diamond, Nature 428, 542 (2004).

[77] Y Takano, M Nagao, I Sakaguchi, M Tachiki, $\mathrm{T}$ Hatano, $\mathrm{K}$ Kobayashi, $\mathrm{H}$ Umezawa, H Kawarada, Superconductivity in diamond thin films well above liquid helium temperature, Appl. Phys. Lett. 85, 2851 (2004).

[78] K Antonowicz, Possible superconductivity at room temperature, Nature 247, 358 (1974).

[79] K Antonowicz, The effect of microwaves on dc current in an Al-Carbon-Al sandwich, Physica Status Solidi A 28, 497 (1975).

[80] N Agrait, J Rodrigo, S Vieira, On the transition from tunneling regime to point-contact: graphite, Ultramicroscopy 42-44, Part 1, 177 (1992).

[81] R Nandkishore, L S Levitov, A V Chubukov, Chiral superconductivity from repulsive interactions in doped graphene., Nat. Phys. 8, 158 (2012).

[82] A M Black-Schaffer, S Doniach, Resonating valence bonds and mean-field d-wave superconductivity in graphite, Phys. Rev. B 75, 134512 (2007).

[83] N B Kopnin, T T Heikkilä, G E Volovik, Hightemperature surface superconductivity in topological flat-band systems, Phys. Rev. B 83, 220503 (2011).

[84] N García, P Esquinazi, Mean field superconductivity approach in two dimensions, J. Supercond. Nov. Magn. 22, 439 (2009).

[85] G Profeta, M Calandra, F Mauri, Phononmediated superconductivity in graphene by lithium deposition, Nat. Phys. 8, 131 (2012).
[86] B Uchoa, A H C Neto, Superconducting states of pure and doped graphene, Phys. Rev. Lett. 98, 146801 (2007).

[87] N B Kopnin, E B Sonin, BCS superconductivity of Dirac electrons in graphene layers, Phys. Rev. Lett. 100, 246808 (2008).

[88] N Reyren, S Thiel, A D Caviglia, L F Kourkoutis, G Hammerl, C Richter, C W Schneider, T Kopp, A S Rüetschia, D Jaccard, M Gabay, D A Muller, J M Triscone, J Mannhart, Superconducting interfaces between insulating oxides, Science 317, 1196 (2007).

[89] A Gozar, G Logvenov, L F Kourkoutis, A T Bollinger, L A Giannuzzi, L A Muller, I Bozovic, High-temperature interface superconductivity between metallic and insulating copper oxides, Nature 455, 782 (2008).

[90] F Muntyanua, A Gilewski, K Nenkov, J Warchulska, A Zaleski, Experimental magnetization evidence for two superconducting phases in Bi bicrystals with large crystallite disorientation angle, Phys. Rev. B 73, 132507 (2006).

[91] F Muntyanua, A Gilewski, K Nenkov, A Zaleski, V Chistol, Superconducting crystallite interfaces with $T_{c}$ up to $21 \mathrm{~K}$ in $\mathrm{Bi}$ and $\mathrm{Bi}$ Sb bicrystals of inclination type, Solid State Commun. 147, 183 (2008).

[92] Y Kopelevich, P Esquinazi, Graphene physics in graphite, Adv. Mater. (Weinheim, Ger.) 19, 4559 (2007).

[93] W A Muñoz, L Covaci, F Peeters, Tightbinding description of intrinsic superconducting correlations in multilayer graphene, Phys. Rev. B 87, 134509 (2013).

[94] Y Kawashima, Possible room temperature superconductivity in conductors obtained by bringing alkanes into contact with a graphite surface, AIP Advances 3, 052132 (2013). 